\title{
Evaluating the effectiveness of different approaches to home support for people in later stage dementia: a protocol for an observational study
}

\section{Dr Helen Chester}

Personal Social Services Research Unit

Division of Population Health

Health Services Research and Primary Care

School of Health Sciences

Faculty of Biology, Medicine and Health

University of Manchester

Manchester Academic Health Science Centre

Manchester, UK

Dr Paul Clarkson

Personal Social Services Research Unit

Division of Population Health, Health Services Research and Primary Care

School of Health Sciences

Faculty of Biology, Medicine and Health

University of Manchester

Manchester Academic Health Science Centre

Manchester, UK

Dr Jane Hughes

Personal Social Services Research Unit

Division of Population Health, Health Services Research and Primary Care School of Health Sciences

Faculty of Biology, Medicine and Health

University of Manchester

Manchester Academic Health Science Centre

Manchester, UK

Professor lan Russell

Swansea Trials Unit

School of Medicine

Swansea University

Swansea, UK

Joan Beresford

Personal Social Services Research Unit

Division of Population Health, Health Services Research and Primary Care School of Health Sciences

Faculty of Biology, Medicine and Health

University of Manchester

Manchester Academic Health Science Centre

Manchester, UK

Professor Linda Davies

Manchester Centre for Health Economics

Division of Population Health, Health Services Research and Primary Care 
School of Health Sciences

Faculty of Biology, Medicine and Health

University of Manchester

Manchester Academic Health Science Centre

Manchester, UK

Dr David Jolley

Personal Social Services Research Unit

Division of Population Health, Health Services Research and Primary Care

School of Health Sciences

Faculty of Biology, Medicine and Health

University of Manchester

Manchester Academic Health Science Centre

Manchester, UK

Dr Julie Peconi

Swansea Trials Unit

School of Medicine

Swansea University

Swansea UK

Professor Fiona Poland

School of Health Sciences

Faculty of Medicine and Health Sciences

University of East Anglia

Norwich, UK

Professor Chris Roberts

Division of Population Health, Health Services Research and Primary Care

School of Health Sciences

Faculty of Biology, Medicine and Health

University of Manchester

Manchester Academic Health Science Centre

Manchester, UK

Caroline Sutcliffe

Personal Social Services Research Unit

Division of Population Health, Health Services Research and Primary Care

School of Health Sciences

Faculty of Biology, Medicine and Health

University of Manchester

Manchester Academic Health Science Centre

Manchester, UK

Professor David Challis

Personal Social Services Research Unit

Division of Population Health, Health Services Research and Primary Care

School of Health Sciences

Faculty of Biology, Medicine and Health

University of Manchester 
Manchester Academic Health Science Centre

Manchester, UK

and members of the HoSt-D (Home Support in Dementia) Programme Management Group

Author for correspondence: Dr Helen Chester, Personal Social Services Research Unit (PSSRU), 2nd Floor Crawford House, University of Manchester, M13 9QS, UK. Telephone number: 01612755345 (Email: helen.chester@manchester.ac.uk) 


\section{Abstract}

Background: Dementia is a major health problem with a growing number of people affected by the condition, both directly and indirectly through caring for someone with dementia. Many live at home but little is known about the range and intensity of the support they receive. Previous studies have mainly reported on discrete services within a single geographical area. This paper presents a protocol for study of different services across several sites in England. The aim is to explore the presence, effects and cost-effectiveness of approaches to home support for people in later stage dementia and their carers.

Methods: This is a prospective observational study employing mixed methods. At least 300 participants (people with dementia and their carers) from geographical areas with demonstrably different ranges of services available for people with dementia will be selected. Within each area, participants will be recruited from a range of services. Participants will be interviewed on two occasions and data will be collected on: their characteristics and circumstances; quality of life; carer health and burden; and informal and formal support for the person with dementia. The structured interviews will also collect qualitative data to explore the perceptions of older people and carers.

Conclusions: This national study will explore the components of appropriate and effective home support for people with late stage dementia and their carers. It aims to inform commissioners and service providers across health and social care.

Key words: dementia; carers; home support; observational study; costeffectiveness; outcomes; quality of life; service receipt

Running title: Home support for people with dementia: a protocol 


\section{Introduction}

Dementia is a progressive disorder leaving individuals less able to care for themselves, more prone to emotional and behavioral problems and more likely to have poor physical health (MacKnight and Rockwood 2001; Burns et al. 2005; Alzheimer's Society 2014). With population ageing dementia represents a major public health and care challenge (Ferri et al. 2005, Alzheimer's Society 2014). About 60 per cent of people with dementia in the United Kingdom live in private households (Knapp et al. 2007). The National Dementia Strategy in England identified helping people to live well with dementia as a priority and acknowledged that non-medical aspects of care influence the experience of patients and carers and the economics of care. For them to live well at home evidence is required about the most appropriate and effective forms of support for them and their carer (Department of Health 2009). Though little is known about the provision, delivery or effectiveness of home support services, it is likely that there is variation across the country because NHS services are designed to promote uniformity whilst diversity is characteristic of local authorities (Wistow, 2012). There has been limited research into the effectiveness of psychosocial and technology-based interventions and different models of home support for people with late stage dementia (Dawson et al., 2015; Low and Fletcher, 2015; Clarkson et al., submitted). This study seeks to fill this gap. 


\section{Investigating home support for people with dementia}

Research in home support for people with dementia raises a number of complex issues. These include the interweaving of formal and informal care; identifying the views of people with dementia themselves; the use of mixed methods, both qualitative and quantitative; and measurement of outcomes and costs. These are considered briefly below.

People with dementia often rely on a combination of formal support by health and social care services and informal support from family and friends. More precise descriptions of the components employed ('who did what, where and how') are needed to evaluate their effectiveness not just in terms of cost but measures of quality of life (Clarkson et al., submitted). As carers provide an essential link between services and the person being cared for, it is important to achieve the right balance between both formal and informal care (Noelker and Bass, 1989; Zarit et al., 1999; Carpentier and Grenier, 2012). Networks of support are important in identifying risk of entry into long-term care (Bowling, 1994). Five network types of informal support from family, friends, neighbours and the community have been identified (Wenger, 1991; Wenger and Tucker, 2002). To be effective in reducing carer burden, support must be appropriate; of sufficient intensity; and target the behaviors and aspects carers find most challenging (Zarit et al., 1999; Venables et al., 2006; Challis et al., 2016).

Eliciting the views of older people in later stage dementia in this process constitutes a challenge. For some their level of impairment means that they lack the capacity to 
participate in interviews. However, research has demonstrated that many can answer questions about their quality of life, even in the presence of significant cognitive deficits (Mozley et al., 1999; Hoe et al., 2005; Moyle et al., 2010). Where this is not possible, complementary proxy measures to capture the quality of life of people with dementia have shown acceptable psychometric properties (Smith et al., 2005; Mulhern et al., 2013). It is important to include these to capture the circumstances of participants who would otherwise have been unable to respond, whilst acknowledging their limitations (Magaziner et al., 1997; Selai and Trimble, 1999; Bryan et al., 2005).

The complexity of dementia research requires a mixed methods approach, synthesizing both quantitative and qualitative data (Pritchard and Dewing, 2001; Johnson et al., 2007; Moyle, 2010; Robinson et al., 2011). By combining the two in a single interview, an embedded ethnographic approach provides a 'sociological lens' to explore the pattern of communications between the person with dementia and their carer whilst completing standard measures (Poland and Birt, 2016 p.771). Elsewhere this has been described as 'ethnography-understood-as-fieldwork' (Brewer, 2000 p.17) based on the premise that embedded research is founded on the principles of immersion fieldwork (Lewis and Russell, 2011). In this study this is undertaken within a more structured data collection.

Objective measurement of outcomes for people with late stage dementia living at home and their carers is complex. However, people with dementia prefer care at home (Challis et al., 2016). Outcomes for carers seek to measure burden and stress but may not detect change (Zarit et al., 1998; Zarit and Leitsch, 2001). Measures of 
cost and cost-effectiveness require data about receipt of health and social care services (Challis et al., 2016). It is also important to collect data relating to the adequacy of services and areas of unmet need (Challis and Davies, 1986).

\section{Aims of study}

This study forms part of a wider research program: 'Effective Home Support in Dementia Care: Components, Impacts and Costs of Tertiary Prevention.' It comprises nine distinct but interrelated projects funded through a National Institute for Health Research (NIHR) Program Grant for Applied Research (DTC-RP-PG0311-12003). The aim of this specific project is to explore the presence and effect of different approaches to home support for people in later stage dementia within selected geographically distinct areas in England. Detailed research questions are:

1) What approaches do home care agencies and other professional staff use to support people in later stage dementia and their carers at home?

2) What are the effects of these different approaches on outcomes for people with later stage dementia and their carers?

3) What is the cost-effectiveness of these different approaches to providing home support?

By home support we mean formal support from services which complements informal support provided by family and friends (described as carers). Categorized according to its expressed purpose, it includes: supportive emotional and practical 
help; education, including skills training in managing behaviour; and more structured therapeutic interventions, such as counselling.

\section{Methods}

This is a prospective observational study using mixed methods (Berger et al., 2012) to study outcomes for older people with dementia and their carers after receiving different components of home support. It measures the relative effectiveness of different approaches to providing support at home. Interviews will collect both quantitative and qualitative data, with the latter exploring the perceptions of older people and carers. Participants (people with dementia and their carers) will be interviewed at home on two occasions, six months apart. The same follow-up period has detected change in similar studies (Challis et al., 2004; Clarkson et al., 2011). Data will be collected primarily from the carer but also from the person with dementia wherever possible. The first interview will occur at a common baseline, when participants have received an assessment or formal review of their care needs. The second interview will occur when they have had services for six months following this assessment in their current living environment. The same interview schedule will be

administered at each time point to facilitate comparison. Domicile of the person with dementia 12 months after the baseline interview will be collected to estimate avoidance of and time to admission to hospital or care home.

\section{Sample}


To cover the different types of support available across the country, a two stage process will be used to recruit participants, defined as people with dementia and their carers. First, sites (geographical areas) will be selected from two national surveys undertaken within the program (PSSRU, 2015). Sites will be stratified into three groups (low 1-8, medium 9-10 and high 11 or more) using sixteen indicators reflecting differences in the range of services available to older people with dementia living at home. Second, at least ten participants will be recruited from each of the following services within each geographical area: home care provider; respite services (day or overnight); community mental health teams; and local carer support services. To this end, each service will be asked to identify from their records a cohort of eligible people in receipt of home support.

A completed sample size of 310 will provide $80 \%$ power to detect a small effect size (Cohen's $d=0.3$ ) at a significance level of $5 \%$. This calculation is based on the regression model which will be use to explore relationships between covariates and outcomes. To take account of attrition between the two surveys, the target sample size at baseline interview is 400 .

\section{Inclusion Criteria}

The target population is people in the later stages of dementia, receiving home support. The inclusion criteria are:

1) Aged 60 years and over;

2) Have an informal carer (who they may or may not live with); 
3) Have received an assessment or review of their care needs within the last three months by a professional acting as a care manager;

4) Considered by professionals to be in the later stages of dementia;

5) Receiving support at home, including supported accommodation (where home care may be part of an integrated care package);

6) Resident within one of the ten sampled geographical areas.

Referrers within health and social care agencies will be advised to use an initial screening question to identify people who are 'very confused' based on earlier research to guide the identification of people with later stage of dementia (Levin et al., 1989).

\section{Study measures}

Table 1 summarizes study measures and provides details of whether they will be completed by the person with dementia or the carer (with the latter sometimes completing a proxy measure on behalf of the former). Data will be collected at two time points, baseline (week 0) and follow-up (week 26), thus measuring change over time. Most are standardized instruments, with demonstrated validity and reliability for people with dementia in the community. Measures fall into three broad groups, those relating to: the person with dementia's characteristics and circumstances; carer characteristics, health and burden; and support to the person with dementia. Data about the support received by people with dementia and their carers will be used in three ways: to identify components of home support; to explore the 
relationship with outcomes (Challis et al., 2016); and to analyse the costeffectiveness of different approaches to home support (Drummond et al., 2005).

[Table 1 around here]

\section{Qualitative component}

Ethnographic data will be collected unobtrusively within a more structured research process at follow-up interviews with a sub-sample of participants (people with dementia and carers) (Lewis and Russell, 2011). This sub-sample will be selected by the Practitioner Assessment of Network Type (PANT) (Wenger, 1991) completed at first interview. For these participants, the structured interviews will be audio-recorded to capture values and experiences around the quantitative component of the study and provide richer contextual and conversational data. A qualitative approach is viewed as a particularly useful means of gaining the perspective of those who are severely cognitively impaired (Cahill and Diaz-Ponce, 2011). The sample size for analysis will be determined according to both pragmatic criteria (resources available, time for analysis) and by data saturation (O'Reilly and Parker, 2012) and is estimated at 30 interviews.

\section{Data security}

Paper questionnaires will be completed by interviewers, stored securely and entered into MACRO4, a secure data capture facility (InferMed Limited, 2015). Participant identification numbers will link data from each interview and ensure that the 
participant remains anonymous in the dataset. Participants' contact details, needed to invite them to take part in interviews, will be kept separately on a passwordprotected computer. All personally identifiable information from transcripts of qualitative interviews will be removed. Data will be stored securely for 10 years following the completion of the study.

\section{Ethical considerations}

Ethical approval has been granted by the National Research Ethics Service (NRES) (15/NW/0822). The interview schedule was designed to minimize participant burden. Participants will be assured that their participation, non-participation or ending their participation mid-interview will not influence their care arrangements.

Informed consent will be sought from participants before starting the research. Staff working within agencies will: introduce the study to participants; provide them with an information sheet; and gain their consent to be contacted by a member of the research team. Before conducting the interview, the researcher will explain the study to participants and obtain their formal consent. Researchers will decide upon the capacity of people with dementia to consent in accordance with the Mental Capacity Act 2005 (House of Commons, 2005). It will be assumed that people with dementia can consent to participate unless there is evidence to the contrary. This decision will be made in conjunction with the carer who will be asked for their opinion on whether or not the person with dementia would want to be involved. If they decide the person with dementia would have no objection to taking part the carer will provide written consent on their behalf. 


\section{Governance arrangements}

An Observational Study Group to oversee and resolve operational issues; a Programme Steering Committee to oversee the entire programme; a Methodology Group responsible for the strategic development and review of methodology and oversight of data collection and analysis for this observational study; and a Public, Patient and Carer Reference Group for the entire programme, notably to review the interview schedules for this study, have been established.

\section{Data analysis}

Data from all participants will be pooled for the purpose of analysis.

Exploratory analysis: descriptive statistics (means, standard deviations for continuous variables and frequencies and percentages for categorical variables) will be calculated for demographic, service receipt and outcome variables to check them before statistical modelling; also bivariate associations amongst variables. The distributions of the outcome measures will be reviewed for normality, and floor and ceiling effects.

Identification of approaches to home care support: To address research question 1, service receipt information will be analysed to describe naturally occurring packages of care received by people with dementia and their carers. Data reduction techniques, factor analysis and cluster analysis (Kline, 1994; Rogerson, 2001), will be used. 
Outcome analyses: To address research question 2, multiple regression will be used to test the relationship between outcome measures and covariates. Within these models the relative effectiveness of each approach to home support will be estimated. To minimize the risk of confounding, that is the systematic error that occurs when there is a failure to account for variables associated with both receipt of a particular care package and the outcomes under study (Trojano et al., 2009), propensity scores will be used. These reduce the effects of baseline characteristics on receipt of different care packages to one composite measure and facilitate adjustment for this in multivariate models (Austin, 2011).

Cost-effectiveness analysis: To address research question 3, a cost-effectiveness analysis of different approaches to home support will be undertaken. National unit costs for specific items of service will be used to estimate the costs of services used. For the primary analysis QALYs will be estimated from the EQ-5D-5L using the recently published value set (Devlin et al., 2016). QALYs gained between baseline and follow-up interview (26 weeks) will be estimated as the number of weeks multiplied by the utility of observed survival. Covariates which may affect the costs or outcomes will be derived from relevant published economic evaluations and discussion with the programme research team (Davies et al., 2008). Costs and QALYs will be bootstrapped to generate pairs of net costs and QALYs. These will then be used to estimate the probability that a model of care may be cost-effective. Cost-effectiveness planes and cost effectiveness acceptability curves (CEACs) will be plotted. Net benefit statistics will be estimated - these approaches require that net QALYs are revalued using a monetary value that reflects decision makers willingness to pay to gain (WTPT) 1 QALY. In line with current estimates of the 
WTPT implicit in The National Institute for Health and Care Excellence decisions we will use the range of £0 to £20k (Raftery, 2014; Claxton et al., 2015). CEACs summarize uncertainty associated with ICERs (Fenwick et al., 2001) in the form of the probability that the approach in question is cost-effective without hypothesis testing or risk of Type II errors, that is failing to detect a real effect.

Sensitivity analysis: This will explore structural uncertainty related to study design decisions. These include re-estimating the results using: high and low sets of unit costs; the crosswalk value set to estimate utility from the EQ-5D-5L (van Hout et al 2012); the DEM-QoL as the measure of health benefit; using the primary and secondary clinical measures (e.g. Bristol Activities of Daily Living Scale, a primary outcome measure); different approaches to missing data (e.g. complete cases only; impute missing observations only); and predictions of costs and QALYs over longer time horizons (e.g. 5 and 10 years).

Qualitative analysis: To provide additional data in respect of research questions 1 and 2, thematic and narrative analysis of interview transcripts will be undertaken to elicit the values, experiences and routines of participants in relation to the topics of data being collected for the main study. At least two researchers will analyse each transcript. All issues will be initially identified, then, through an iterative process and developing conceptual abstraction, the major themes and processes will be identified. To establish when the ethnographic interviews should cease, analysis will be on a rolling basis (Guest et al. 2006).

\section{Discussion}


The changing global demographics of dementia have led to worldwide predictions of unaffordable treatment and care costs over the coming decades (Rossor and Knapp, 2015). In England, the cost of care is growing (Department of Health, 2012) and there is a need for research to span the artificial divide in health and social care (Alzheimer's Society, 2014). This study seeks to provide evidence to inform this debate by collecting data on the well-being of service users and carers and the use and cost of health and social care.

Previous studies have investigated home support for people with dementia and their carers by single services in single areas (e.g. Rothera et al., 2008) or investigated the effectiveness of a single form of treatment (e.g. Orgeta et al., 2015). In contrast the current study collects data from multiple sites about home support for people with dementia and aims to investigate the effectiveness of the mix of several services. It builds on previous work undertaken by the research team into intensive case management for older people with dementia at risk of admission to residential or nursing care and a pilot project which investigated existing models of generic and specialist home care for people with dementia in England (Challis et al., 2002; 2016; 2010).

This study is novel in three aspects:

1. First, people with dementia and their carers will be recruited from geographical areas known to exhibit differences in the range of available services. Areas will be identified through national surveys of health and social care, another study within the 
program. This will permit comparisons between areas with different ranges of services and individual experiences.

2. Second, building on a systematic literature review within the program (Clarkson et al., 2016), patterns of service receipt will be examined to seek to identify service clusters (types of service mix) and their associated outcomes for carers and people with dementia and their cost-effectiveness.

3. Third, a sub-set of structured interviews will be audio-recorded to explore the values and experiences of participants alongside the structured data collection (Lewis and Russell, 2011). This will also compare the influence of different support networks on community care (Wenger and Tucker, 2002).

\section{Feasibility}

Four methodological choices were made in the design of this study, the first two of which may be perceived as limitations.

1. First, a prospective observational approach was chosen. As care is purchased and provided by local government, the National Health Service, voluntary organisations, private providers and people themselves and varies both within and between areas, this is in effect a natural experiment. It is hoped it will yield empirical evidence on promising forms of care to inform an appropriate intervention for evaluation in a future trial. 
2. Second, to study participants receiving a range of services a two stage process was adopted: the services available across geographical areas were categorized using data from national surveys of health and social care provision conducted within the programme and a stratified sample was drawn; and within these areas participants were selected from a variety of services - community mental health teams, home care agencies, respite care and services for carers. To facilitate the identification of eligible participants, referrers within health and social care agencies will be advised to use an initial screening question to guide the identification of people with later stage dementia (Levin et al., 1989; Moriarty and Webb, 2000).

3. Third, to yield a powerful completed dataset of at least 300 participants, three strategies were adopted. To allow for attrition, 400 participants will be recruited at baseline. Interview schedules were designed in conjunction with a panel of lay advisers to ensure they use validated measures whenever possible and to minimize participant burden and dropout. It has been arranged to interview participants interviewed at baseline who move location prior to follow-up whenever possible.

4. Fourth, MACRO4, an electronic data management system (InferMed Limited, 2015), will be used to monitor participants' progress through the study and maintain a full audit trail, notably of all data amendments with reason and time. This study will be managed in collaboration with the Swansea Trials Unit.

Thus the study aims to provide commissioners and service providers with valuable information about the components of appropriate and effective home support for people with dementia and their carers across the health and social care sectors. 


\section{Conflict of interest}

None.

\section{Description of authors' roles}

H. Chester led the drafting, review and revision of the protocol and drafted the manuscript. P. Clarkson conceived the study, and drafted and reviewed the protocol. J. Hughes conceived the study and participated in the review and revision of the protocol and drafted the manuscript. C. Sutcliffe, the study manager, participated in the review and revision of the protocol. L. Davies conceived the study, especially the economic component and participated in the drafting and revision of the protocol. C. Roberts and I. Russell conceived the study, especially the sample design and statistical analysis and participated in the drafting and revision of the protocol. F. Poland conceived the study, especially the qualitative component. J. Beresford, D. Jolley and J. Peconi participated in the review and revision of the protocol. D. Challis conceived the study and participated in the review and revision of the protocol. All authors critically reviewed this paper and approved the final manuscript.

\section{Acknowledgements}

We thank the health service and local provider organisations who have agreed to take part in the research by acting as sites for recruitment of participants. In particular we thank the staff of Greater Manchester National Institute for Health 
Research (NIHR) Clinical Research Network. Other members of the Programme Management Group are: Carol Ainsworth and Reagan Blyth (Pennine Care NHS Foundation Trust); Narinder Kapur (University College London); Martin Orrell (Nottingham University); Brenda Roe (Edge Hill University); and Jean Tottie (Uniting Carers).

This project was funded by the National Institute for Health Research (NIHR) under its Programme Grants for Applied Research (Grant reference number: DTC-RP-PG0311-12003). The views expressed are those of the authors and not necessarily those of the NIHR or the Department of Health. 


\section{References}

Alzheimer's Society. (2014). Dementia UK: 2014 edition. London: Alzheimer's Society.

Austin, P.C. (2011). An introduction to propensity score methods for reducing the effects of confounding in observational studies. Multivariate Behavioral Research, 46, 3, 399-424.

Beecham J.K. and Knapp, M.R.J. (1992). Costing psychiatric interventions, in Thornicroft G, Brewin C, Wing JK. (eds.) Measuring Mental Health Needs (pp. 200-224). London: Gaskell.

Berger, M.L., Dreyer, N., Anderson, F., Towse, M.A., Sedrakyan, A. and Normand, S-L. (2012). Prospective observational studies to assess comparative effectiveness: The ISPOR good research practices task force report. Value in Health, 15, 2, 217-230.

Bowling, A. (1994). Social networks and social support among older people and implications for emotional well-being and psychiatric morbidity. International Review of Psychiatry, 6, 41-58.

Brewer, JD. (2000). Ethnography. Buckingham: Open University Press.

Bryan, S., Hardyman, W., Bentham, P., Buckley, A and Laight, A. (2005). Proxy completion of EQ-5D in patients with dementia. Quality of Life Research, 14, 107118.

Bucks, R.S., Ashworth, D.L., Wilcock, G.K. and Siegfried, K. (1996). Assessment of activities of daily living in dementia: development of the Bristol Activities of Daily Living Scale. Age and Ageing, 25, 2, 113-120.

Burns, A., O'Brien, J. and Ames, D. (2005). Dementia. Third edition. London: Hodder Arnold.

Cahill, S. and Diaz-Ponce, A. (2011). "I hate having nobody here. I'd like to know where they all are": can qualitative research detect differences in quality of life among nursing home residents with different levels of cognitive impairment? Aging and Mental Health, 15, 562-572.

Carpentier, N. and Grenier, A. (2012) Successful linkage between formal and informal care systems: the mobilization of help by caregivers of persons with Alzheimer's disease. Qualitative Health Research, 22, 10, 1330-1344.

Challis, D. and Davies, B. (1986). Case Management in Community Care. Aldershot: Gower.

Challis, D., Chesterman, R., Luckett, R., Stewart, K. and Chessum, R. (2002). Care Management in Social and Primary Health Care: The Gateshead Community Care Scheme. Aldershot: Ashgate. 
Challis, D. et al. (2010). Community Support Services for People with Dementia: The Relative Costs and Benefits of Specialist and Generic Domiciliary Care Services. Discussion Paper M245-3. Manchester: PSSRU.

Challis, D. et al. (2004). The value of specialist clinical assessment of older people prior to entry to care homes. Age and Ageing, 33, 1, 25-34.

Challis, D., Sutcliffe, C., Hughes, J., von Abendorff, R., Brown, P. and Chesterman, J. (2016). Supporting People with Dementia at Home. London: Routledge.

Clarkson, P., Brand, C., Hughes, J. and Challis, D. (2011). Integrating assessments of older people: examining evidence and impact from a randomized controlled trial. Age and Ageing, 40, 3, 388-391.

Clarkson, P. et al. (2016). A protocol for a systematic review of effective home support to people with dementia and their carers: components and impacts. Journal of Advanced Nursing, 72, 1, 186-196.

Clarkson, P. et al. (submitted). Overview of systematic reviews: Effective Home Support in Dementia Care, components and impacts - Stage 1, psychosocial interventions for dementia, Journal of Advanced Nursing.

Claxton, K. et al. (2015). Methods for the estimation of the National Institute for Health and Care Excellence cost-effectiveness threshold. Health Technology Assessment, 19, 14.

Dawson, A., Bowes, A., Kelly, F., Velzke, K. and Ward, R. (2015). Evidence of what works to support and sustain care at home for people with dementia: a literature review with a systematic approach, BMC Geriatrics, 15, 59, DOI 10.1186/s12877-015-0053-9.

Davies, L.M. et al. (2008). A randomized trial of the cost-utility of second-generation antipsychotics in people with psychosis and eligible for Clozapine. Value in Health, 11, 4, 549-562.

Department of Health. (2009). Living Well with Dementia: A National Dementia Strategy. London: Department of Health.

Department of Health. (2012). Prime Minister's Challenge on Dementia: Delivering Major Improvements in Dementia Care and Research by 2015. London: Department of Health.

Devlin, N., Shah. K. Feng, Y., Mulhern, B. and van Hout, B. (2016). Valuing Health-Related Quality of Life: An EQ-5D-5L Value Set for England. Office of Health Economics Research Paper 16/01. London: Office of Health Economics.

Drummond, M., Sculpher, M., Torrance, G., O'Brien, B. and Stoddart, G. (2005). Methods for the Economic Evaluation of Health Care Programmes, $3^{\text {rd }}$ Edition. Oxford: Oxford University Press. 
Fenwick, E., Claxton, K. and Sculpher, M. (2001). Representing uncertainty: the role of cost-effectiveness acceptability curves. Health Economics, 10, 8, 779-787.

Ferri, C.P, Prince, M., Brayne, C., Brodaty, H., Fratiglioni, L., Ganguli, M. et al (2005). Global prevalence of dementia: a Delphi consensus study. The Lancet, $366,9503,2112-17$.

Goldberg, D.P. (1978). Manual of the General Health Questionnaire. Windsor: NFER-Nelson.

Guest, G., Bruce, A. and Johnson, L. (2006). How many interviews are enough? An experiment with data saturation and variability. Field Methods, 18, 1, 59-82.

Herdman, M. et al. (2011). Development and preliminary testing of the new fivelevel version of EQ-5D (EQ-5D-5L). Quality of Life Research, 20, 10, 1727-1736.

Hoe, J., Katona, C., Roch, B. and Livingston, G. (2005). Use of the QOL-AD for measuring quality of life in people with severe dementia - the LASER-AD study, Age and Ageing, 34, 130-135.

House of Commons. (2005). Mental Capacity Act 2005, Chapter 9. London: House of Commons.

InferMed Limited. (2015). MACRO Electronic Data Capture: Advanced Data Collection for Clinical Research. http://www.infermed.com/EDC-in-ClinicalResearch/Overview.aspx [accessed 02/02/2015].

Johnson, R. B., Onwuegbuzie, A. J., and Turner, L. A. (2007). Toward a definition of mixed methods research. Journal of Mixed Methods Research, 1, 2, 112-133.

Kline, P. (1994). An Easy Guide to Factor Analysis. London: Routledge.

Knapp, M. et al. (2007). Dementia UK. London: PSSRU, London School of Economics and the Institute of Psychiatry for the Alzheimer's Society.

Levin, E., Sinclair, I. and Gorbach, P. (1989). Families Confusion and Old Age. Aldershot: Gower.

Lewis, S.J. and Russell, A.J. (2011). Being embedded: A way forward for ethnographic research. Ethnography, 12, 3, 398-416.

Low, L-F. and Fletcher, J. (2015). Models of home care services for persons with dementia: a narrative review. International Psychogeriatrics, 27, Special Issue 10, 1593-1600 doi:10.1017/S1041610215000137.

MacKnight, C. and Rockwood, K. (2001). Use of the Chronic Disease Score to measure co-morbidity in the Canadian study of Healthy Ageing. International Psychogeriatrics, 13, Supplement 1, 137-142. 
Magaziner, J. (1997). Use of proxies to measure health and functional outcomes in effectiveness research in persons with Alzheimer disease and related disorders. Alzheimer Disease and Associated Disorders, 11, Suppl. 6, 168-174.

Molloy, D. and Standish, T. (1997). Mental status and neuropsychological assessment: a guide to the Standardised Mini-Mental State Examination. International Psychogeriatrics, 9, 1, 87-94.

Moriarty, J. and Webb, S. (2000). Part of their Lives: Community Care for Older People with Dementia. Bristol: Policy Press.

Moyle, W. (2010). Is quality of life being compromised in people with dementia in long-term care? International Journal of Older People Nursing, 5, 245-252.

Mozley, C. et al. (1999). 'Not knowing where I am doesn't mean I don't know what I like': cognitive impairment and quality of life responses in elderly people. International Journal of Geriatric Psychiatry, 14, 9, 776-783 DOI:10.1002/(SICl)1099-1166(199909)14:9<776::AID-GPS13>3.0.CO;2-C.

Mulhern, B. et al. (2013). Development of DEMQOL-U and DEMQOL-PROXY-U: generation of preference-based indices from DEMQOL and DEMQOL-PROXY for use in economic evaluation, Health Technology Assessment, 17, 1-140.

Noelker L. and Bass, D. (1989). Home care for elderly persons: Linkages between formal and informal caregivers. Journal of Gerontology: Social Sciences, 44, S63S70.

O'Reilly, M. and Parker, N. (2013). Unsatisfactory saturation: a critical exploration of the notion of saturated sample sizes in qualitative research. Qualitative Research, 13, 2, 190-197.

Orgeta, V. et al. (2015). Individual cognitive stimulation therapy for dementia: a clinical effectiveness and cost-effectiveness pragmatic, multicentre, randomized controlled trial. Health Technology Assessment, 19, 64, 1-108.

Personal Social Services Research Unit (PSSRU). (2015). Effective home support in dementia care: components, impacts and costs of tertiary prevention. A five year research programme. Manchester: PSSRU. http://sites.nursing.manchester.ac.uk/pssru/research/DementiaHomeCare/Newslet ters/Newsletter Year2version2.pdf [Accessed 30/08/2016]

Poland, F. and Birt, L. (2016). The agentic person: shifting the focus of care. Aging and Mental Health, 20, 8, 771-772, DOI: 10.1080/13607863.2016.1146873.

Pritchard, E. and Dewing, J. (2001). A multi-method evaluation of an independent dementia care service and its approach. Ageing and Mental Health, 5, 63-72.

Raftery, J. (2014). NICE's Cost-Effectiveness Range: Should it be Lowered? PharmacoEconomics, 32, 7, 613-615. 
Robinson, A. et al. (2011). Mixed methods data collection in dementia research: a "progressive engagement" approach. Journal of Mixed Methods Research, 5, 4, 330-344.

Rogerson, P. (2001). Data Reduction: Factor Analysis and Cluster Analysis, in Rogerson, P. (ed.) Statistical Methods for Geography. London: SAGE Publications.

Rossor, M. and Knapp, M. (2015). Can we model a cognitive footprint of interventions and policies to help meet the global challenge of dementia? The Lancet, 386, 9997, 1008-1010.

Rothera, I. et al. (2008). An evaluation of a specialist multiagency home support service for older people with dementia using qualitative methods. International Journal of Geriatric Psychiatry, 23, 1, 65-72.

Selai, C. and Trimble, M. (1999). Assessing quality of life in dementia. Ageing and Mental Health, 3, 2, 101-111.

Smith, S.C. et al. (2005). Measurement of health-related quality of life for people with dementia: development of a new instrument (DEMQOL) and an evaluation of current methodology. Health Technology Assessment 2005; 9, 10, 1-93.

Trojano, M., Pellegrini, F., Paolicelli, D., Fuiani, A. and Di Renzo, V. (2009). Observational studies: propensity score analysis of non-randomized data. The International MS Journal, 16, 3, 90-97.

van Hout, B., Janssen, M.F., Feng, Y-S., KohImann, T., Busschbach, J., Golicki, D. et al. (2012). Interim scoring for the EQ-5D-5L: Mapping the EQ-5D-5L to EQ5D-3L value sets. Value in Health, 15, 5, 708-715.

Venables, D., Reilly, S., Challis, D., Hughes, J. and Abendstern, M. (2006). Standards of care in home care services: a comparison of specialist and generic services for older people with dementia. Aging and Mental Health, 10, 187-194.

Vernooji-Dassen, M., Felling, A., Brummelkamp, E., Dauzenberg, M., van den Bos, G. and Grol, R. (1999). Assessment of Caregiver's Competence in dealing with the burden of caregiving for a dementia patient: a short sense of competence questionnaire (SSCQ) suitable for clinical practice. Journal of the American Geriatrics Society, 47, 256-257.

Wenger, C. (1991) A network typology: from theory to practice, Journal of Aging Studies, 5, 2, 147-62.

Wenger, G. and Tucker, I. (2002) Using network variation in practice: identification of support network type, Health and Social Care in the Community, 10, 1, 28-35.

Wistow, G. (2012). Still a fine mess? Local government and the NHS 1962 to 2012. Journal of Integrated Care, 20, 2, 101-114. 
Zarit, S. and Leitsch, S. (2001). Developing and evaluating community based intervention programs for Alzheimer's patients and their caregivers. Aging and Mental Health, 5, 1, S84-S98.

Zarit, S.H, Reever, K.E. and Bach-Peterson, J. (1980). Relatives of the impaired elderly: Correlates of feelings of burden. The Gerontologist, 20, 649-655.

Zarit, S., Stephens, M., Townsend, A. and Greene, R. (1998). Stress reduction for family caregivers: effects of adult day care use. Journal of Gerontological Social Science, 53B, S267-S277.

Zarit, S., Gaugler, J. and Jarrott, S. (1999). Useful services for families: research findings and directions. International Journal of Geriatric Psychiatry, 14, 165-181. 
Table 1. Study Measures

\begin{tabular}{|c|c|}
\hline MEASURE & COMPLETED BY \\
\hline \multicolumn{2}{|l|}{ PERSON WITH DEMENTIA CHARACTERISTICS AND CIRCUMSTANCES } \\
\hline Demographic information (including gender, age, ethnicity, marital status) & Carer \\
\hline Living situation (home, hospital, or care home) & Carer \\
\hline Practitioner Assessment of Network Type (PANT) (Wenger and Tucker, 2002) & Carer \\
\hline $\begin{array}{l}\text { Standardized Mini-Mental State Examination (SMMSE) (Molloy and Standish, } \\
1997)^{1}\end{array}$ & PwD \\
\hline Bristol Activities of Daily Living Scale (BADLS) (Bucks et al., 1996) ${ }^{2}$ & Carer \\
\hline \multicolumn{2}{|l|}{ PERSON WITH DEMENTIA QUALITY OF LIFE } \\
\hline $\begin{array}{l}\text { Dementia Quality of Life scale (DEMQOL) - self-report version (29-item) (Smith } \\
\text { et al., 2005) }{ }^{2}\end{array}$ & PwD \\
\hline $\begin{array}{l}\text { Dementia Quality of Life scale (DEMQOL) - proxy version (30-item) (Smith et } \\
\text { al., 2005) } 2,3\end{array}$ & Carer \\
\hline \multicolumn{2}{|l|}{ CARER CHARACTERISTICS, HEALTH AND BURDEN } \\
\hline Demographic information (including gender, age, ethnicity, marital status) & Carer \\
\hline EQ-5D-5L (Herdman et al., 2011) ${ }^{1,3}$ & Carer \\
\hline General Health Questionnaire (GHQ-12) (Goldberg, 1978) $)^{1}$ & Carer \\
\hline $\begin{array}{l}\text { Short Sense of Competence Questionnaire (SSCQ) (Vernooji-Dassen et al., } \\
\text { 1999). }{ }^{\text {. }}\end{array}$ & Carer \\
\hline Zarit Burden Interview (22-item) (Zarit et al., 1980) ${ }^{1}$ & Carer \\
\hline \multicolumn{2}{|l|}{ SUPPORT TO PERSON WITH DEMENTIA } \\
\hline $\begin{array}{l}\text { Informal support by carer (relationship to PwD; care tasks undertaken - nature } \\
\text { and frequency; employment status) }\end{array}$ & Carer \\
\hline $\begin{array}{l}\text { Formal care (health and social care services; adaptations and equipment; } \\
\text { inpatient and outpatient care; ambulance use) }{ }^{4}\end{array}$ & Carer \\
\hline $\begin{array}{l}\text { Reliability, sufficiency and effectiveness of care (personal; daily household; } \\
\text { weekly household) (Challis and Davies, 1986). }{ }^{1}\end{array}$ & Carer \\
\hline
\end{tabular}

$\mathrm{PwD}=$ person with dementia.

1Secondary outcome.

${ }^{2}$ Primary outcome.

${ }^{3}$ For cost-effectiveness analysis

${ }^{4}$ Adapted from Beecham and Knapp (1992). 